\title{
Research Paper \\ Relationship Between Meaning of Life and Spiritual Well-being in the Older People Resid- ing in Nursing Homes Shemiranat, 2014
}

\author{
Shadi Abedi ${ }^{1}$, ${ }^{*}$ Mahshid Foroughan ${ }^{2}$, Mohammad Saeed Khanjani ${ }^{3}$, Enayat Allah Bakhshi ${ }^{4}$, Akram Farhadi ${ }^{2}$
}

1. Department of Rehabmanagment, University of Social Welfare and Rehabilitation Sciences, Tehran, Iran.

2. Iranian Research Center on Ageing, University of Social Welfare and Rehabilitation Sciences, Tehran, Iran.

3. Department of Consulting, University of Social Welfare and Rehabilitation Sciences, Tehran, Iran.

4. Department of Biostatistics, University of Social Welfare and Rehabilitation Sciences, Tehran, Iran.

Crtatt on: Abedi Sh, Foroughan M, Khanjani MS, Bakhshi EA, Farhadi A. [Relationship Between Meaning of Life and Spiritual Well-being in the Older People Residing in Nursing Homes Shemiranat, 2014 (Persian)]. Iranian Journal of Ageing. 2016; 11(3):456-465. http://dx.doi.org/10.21859/sija-1103456

doi : : http://dx.doi.org/10.21859/sija-1103456

Received: 26 Apr. 2016 Accepted: 12 Aug. 2016

Key words: Spiritual well-being, Meaning of life, Older adult, Nursing home

\section{ABSTRACT}

Objectives Spiritual health is one of the significant dimensions of health. It plays an important role in the health of older people and brings aims and meanings to their lives. This study aimed to investigate the relationship between life meaning and spiritual health of elderly people.

Methods \& Materials In this descriptive-analytic study, 204 elderly people living in nursing homes of Shemiranat in 2014 were selected by convenient sampling. Information was collected through Polotzin and Elison's spiritual Well-Being Questionnaires. SPSS software, Pearson correlation coefficient, and tindependent tests were used to analyze the data.

Results The mean scores of spiritual health and life meaning were $72.96 \pm 12.65$ and $53.03 \pm 3.93$, respectively. There were positive and significant relationships between overall spiritual health and life meaning $(P<0.05 ; r=0.31)$, existential health and life meaning $(P<0.05 ; r=0.16)$, and religious health and life meaning $(P<0.05 ; r=0.15)$. There was also a significant relationship between education and life meaning $(P<0.05 ; r=0.12)$.

Conclusion Research on factors associated with the meaning of life and effective interventions to raise it in older adults is recommended.

\section{* Corresponding Author:}

Mahshid Foroughan, PhD

Address: Iranian Research Center on Ageing, University of Social Welfare and Rehabilitation Sciences, Koodakyar Ave., Daneshjoo Blvd., Evin, Tehran, Iran. Tel: +98 (21) 22180077

E-mail: m_foroughan@yahoo.com 


\section{بررسى رابطه معناى زندكى و سلامت معنوى در سالمندان مقيم آسايشكَاهاى شهرستان شميرانات، بروسا}

شادى عابدى'، "مهشيد فروغان"، محمدسعيد خانجانى"، عنايتالله بخشى ?؛ اكرم فرهادى"

|- اكروه مديريت توانبخشى، دانشعاه علوم بهزيستى و توانبخشى، تهران، ايران.

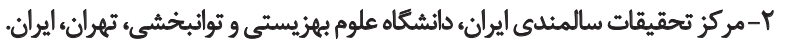

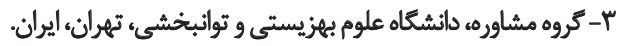

f- Fروه آمار زيستى، دانشكاه علوم بهزيستى و توانبخشى، تهران، ايران.

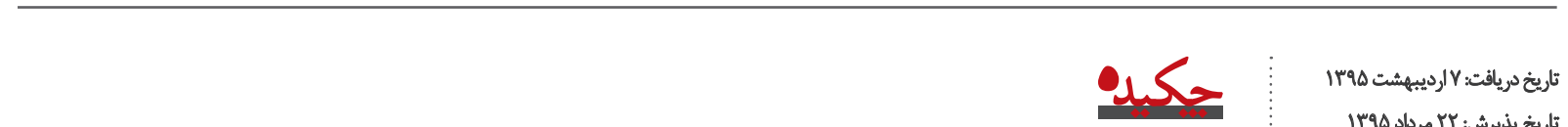

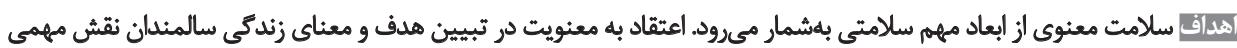

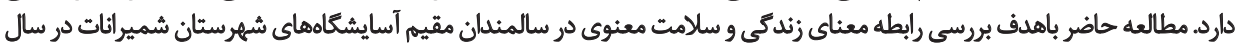

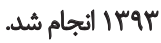

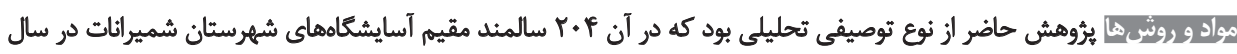

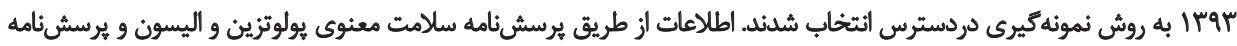

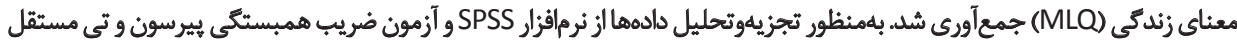

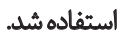

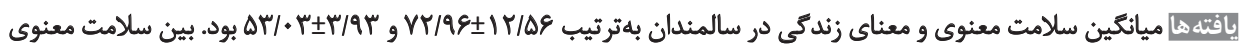

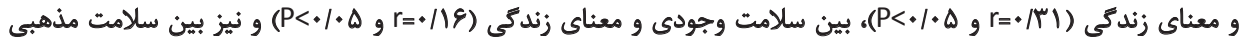

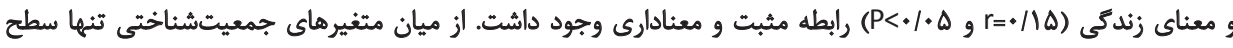

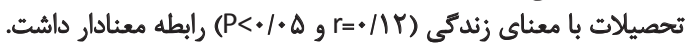

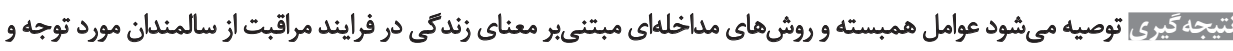

انجام شد، حدود N/ درصد جمعيت كشور را سالمندان تشكيل

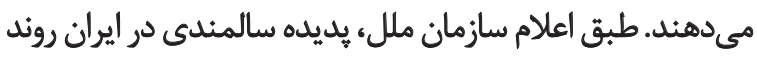

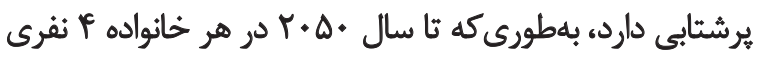

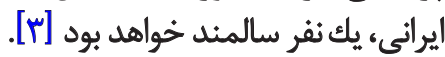
از جمله مسائل روانشناختى كه ممكن است سالمندان با آن

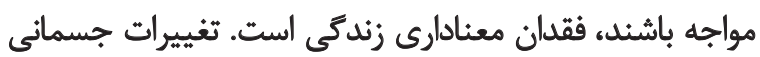

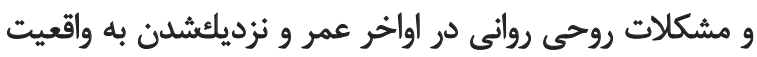

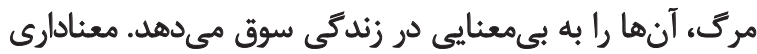

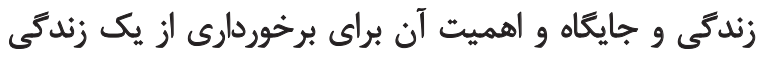

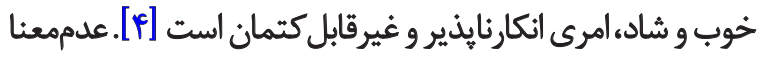

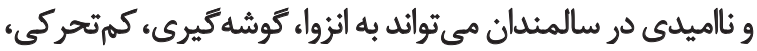

$$
\text { افسردكى و... منجر شود [هان]. }
$$

\section{doles}

سالمندى فرايندى همعانى است كه از نقطه لقاح آغاز مى مئود

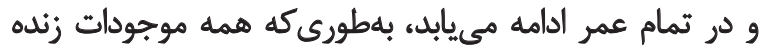

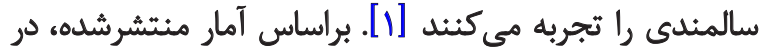

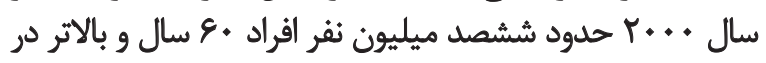

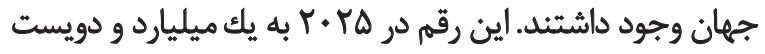

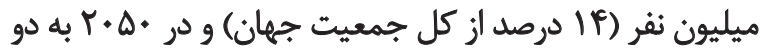

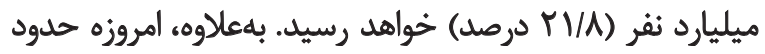

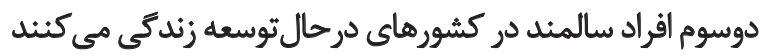

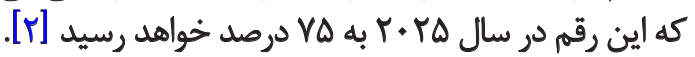

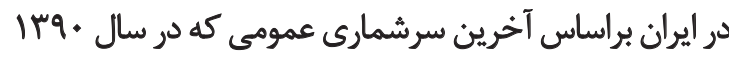




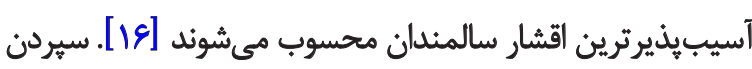

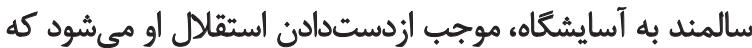

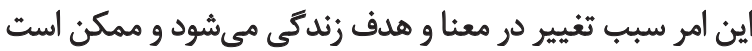

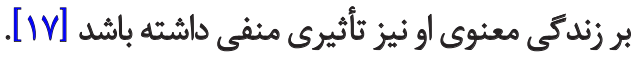

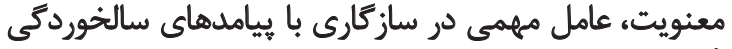

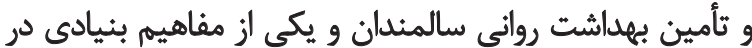

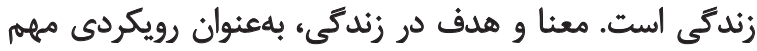

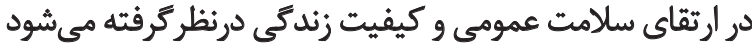

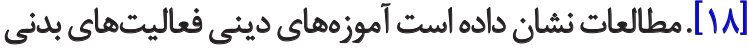

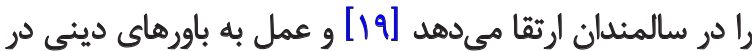

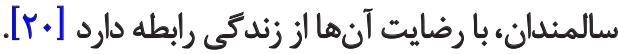

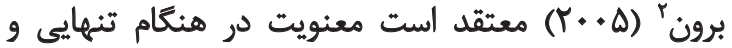

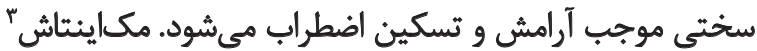

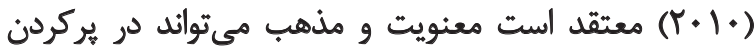

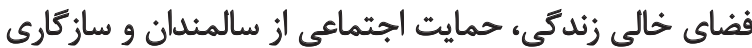

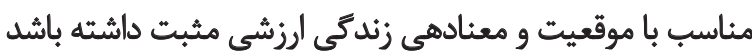

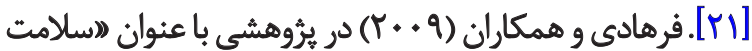

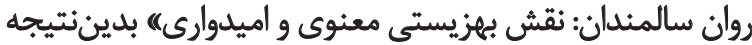

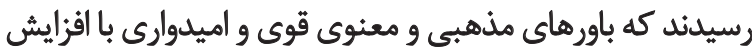

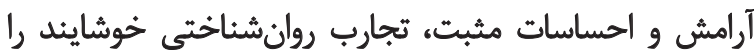

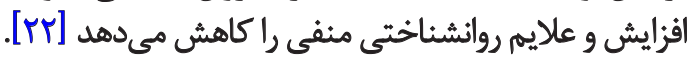

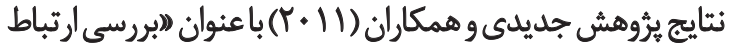

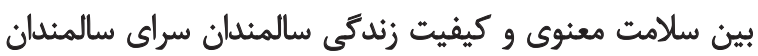

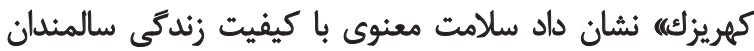

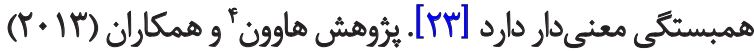

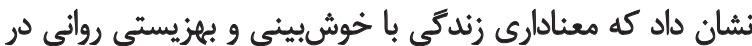

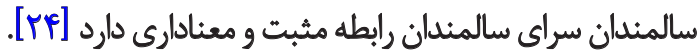

در برخى از مطالعات نيز به بررسى عوامل مؤثر بر معناي

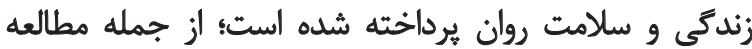

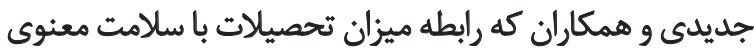

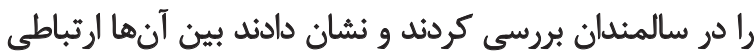

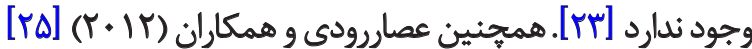

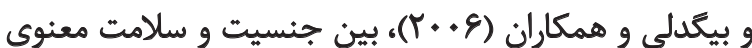

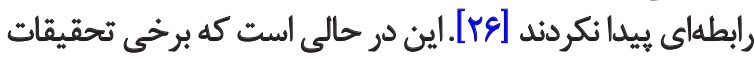

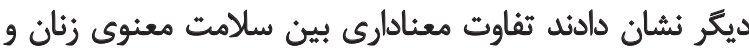

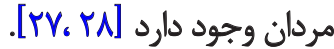

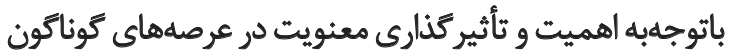

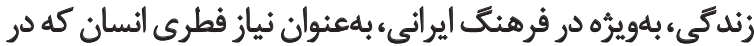

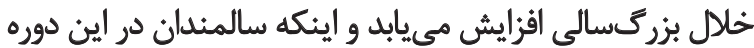

\section{Brown}

3. McIntosh

4. Haewon
دغُدغه معناو دستيابى به زندكى معنادار يكى از اساسىترين

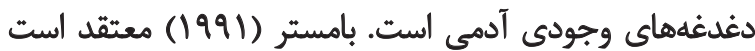

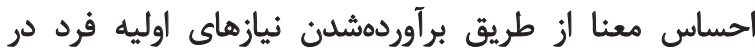

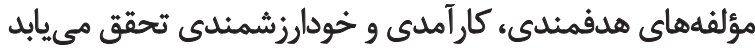

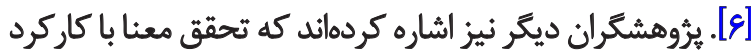

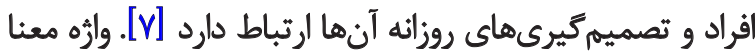

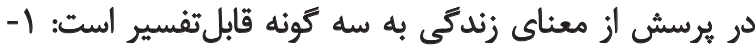

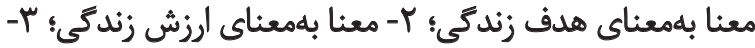

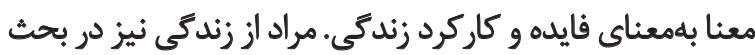

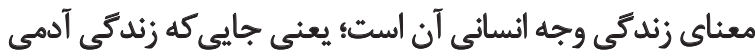

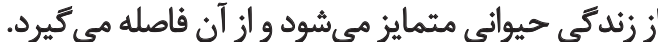

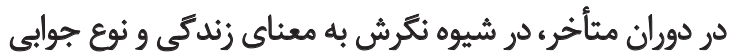

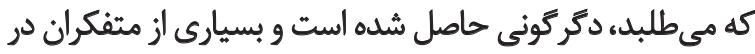

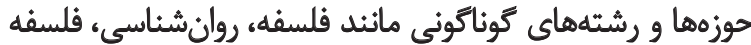

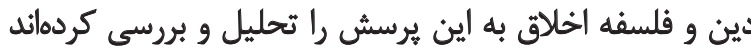

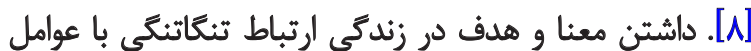

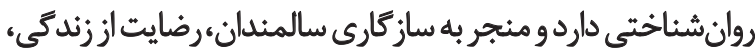

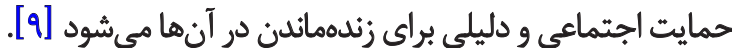
با افزايش سن، تعداد زيادى از سالمندان براى كنترل

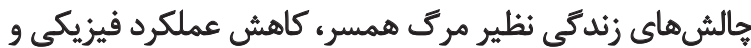

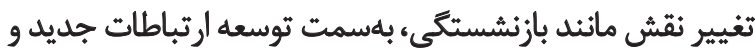

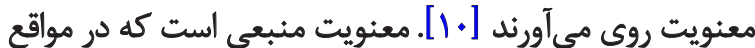

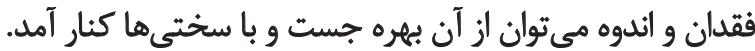

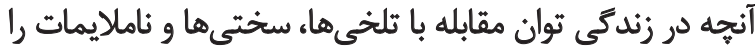

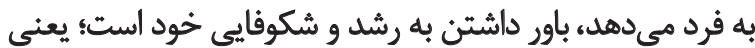

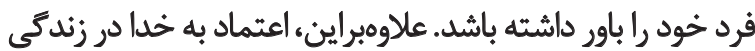

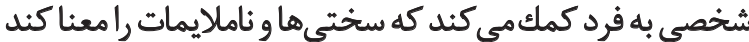

و آنها را سكوى يرش به سوى معنويت بدائد [III]

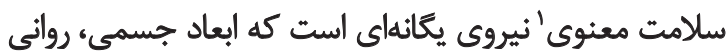

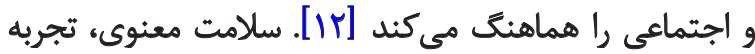

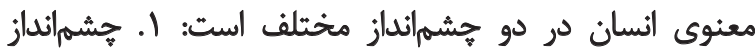

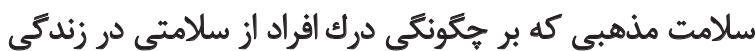

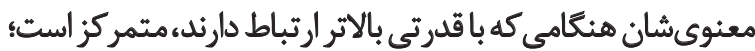

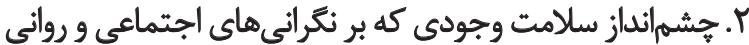

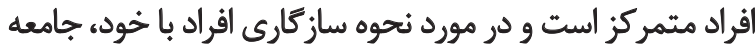

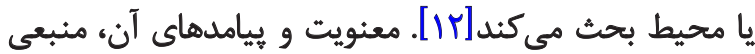

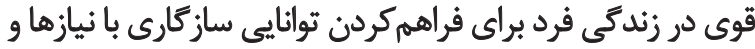

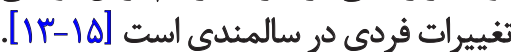

هرجند توجه به سلامت همه سالمندان يكى از مشكلات

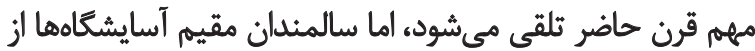
ولويت بيشترى برخوردارند؛ جراكه سالمندان مقيم آسايشكاه جزو المانيان

1. Spiritual health 


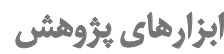

ابزارهاى كردآورى دادهها را يرسشنامه جمعيتشناختى

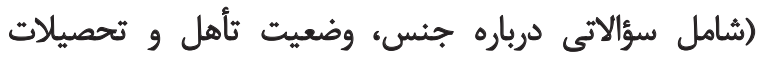

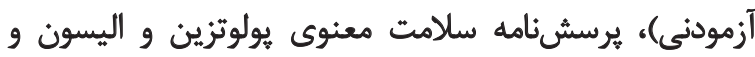

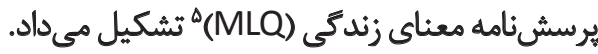

يوسش نامه سالامت معنوى

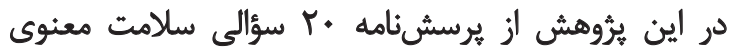

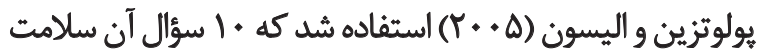

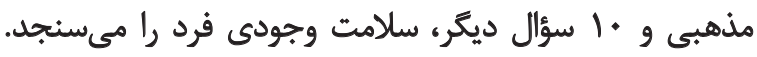

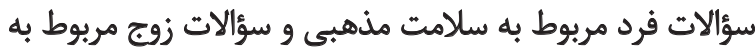

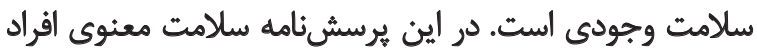

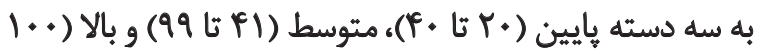

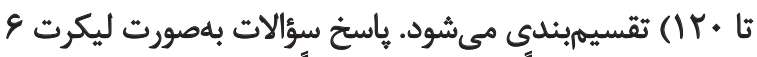

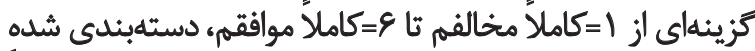

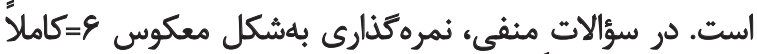

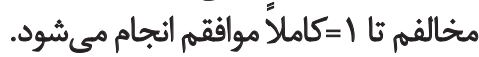

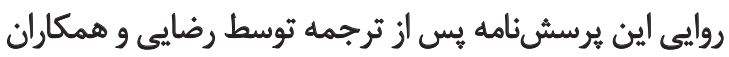

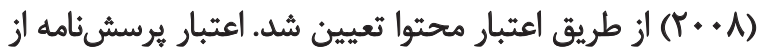

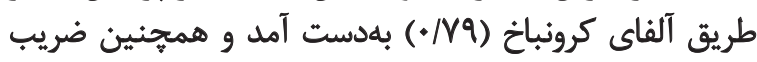

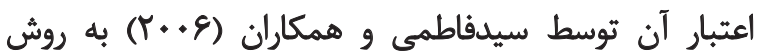

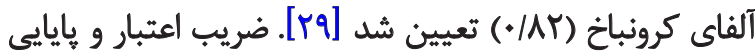

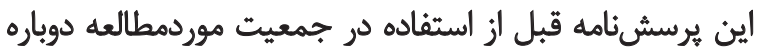

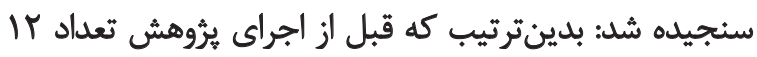

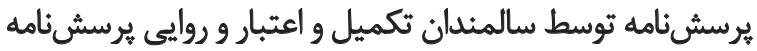

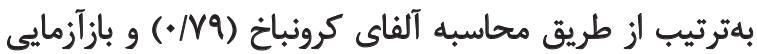

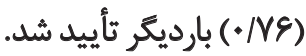

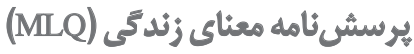

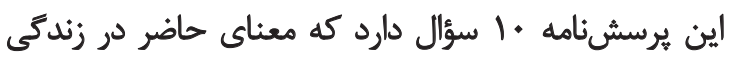

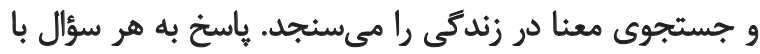

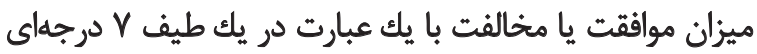

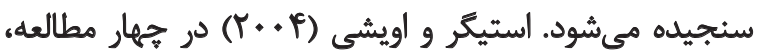

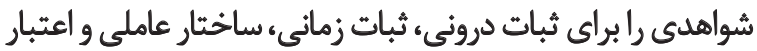

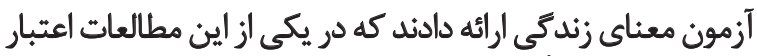

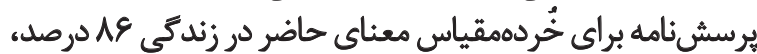

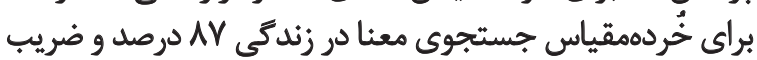

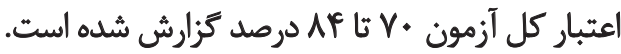

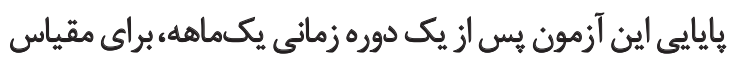

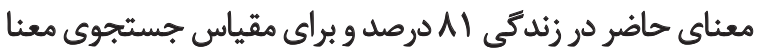

5. Meaning in life Questioner (MLQ)

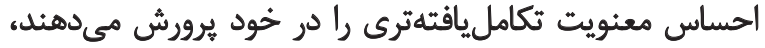

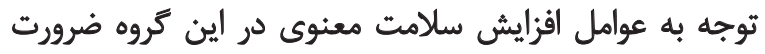

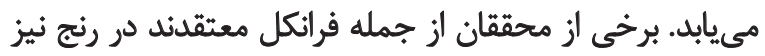

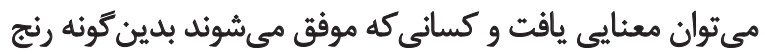

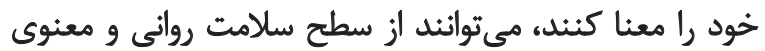

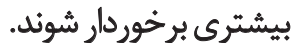

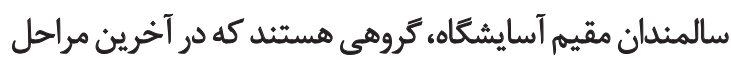

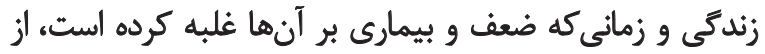

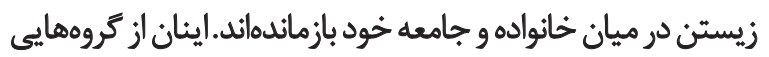

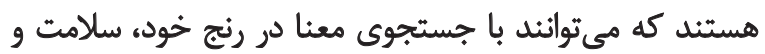
كيفيت زندكى

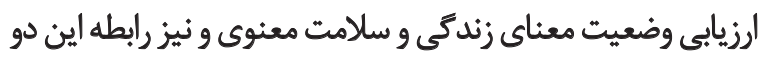

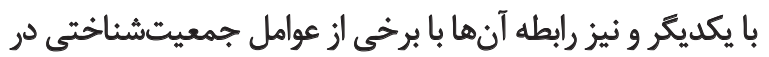
سالمندان مقيم آسايشكاههاى شميرانات أنجام شد.

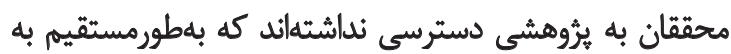

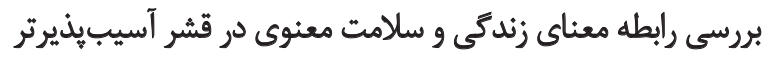

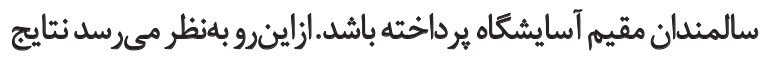

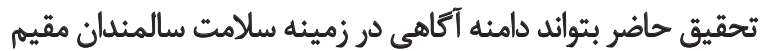

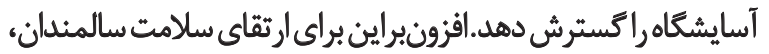

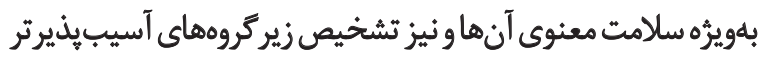

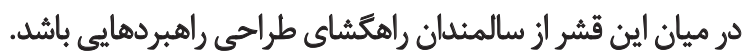

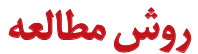

اين مطالعه مقطعى از نوع همبستَّى بود كه در سه ماهله آخر

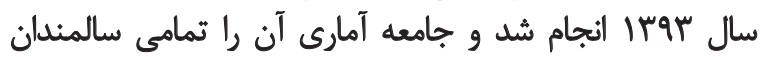

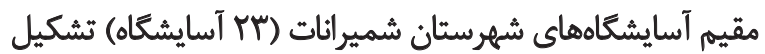

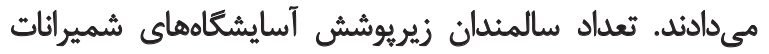

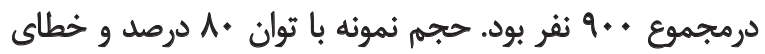

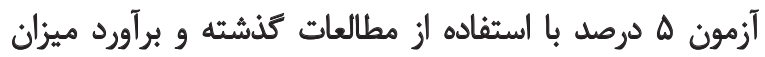

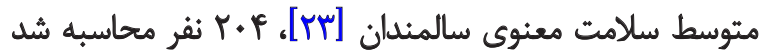

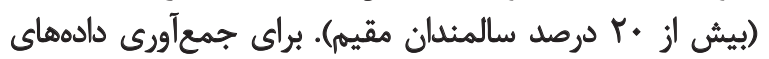

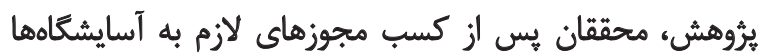

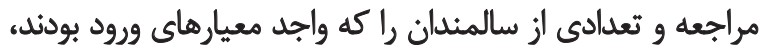
باتوجهبه جمعيت زيريوشش آن مركز انتخاب و وارد مطالعه كردند.

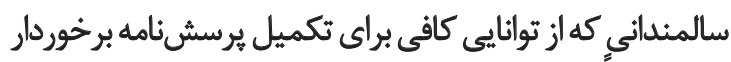

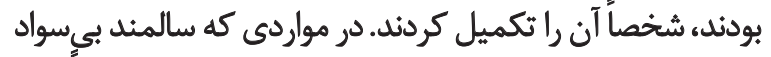

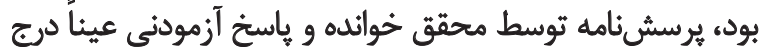

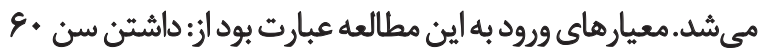

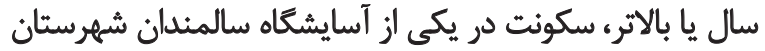

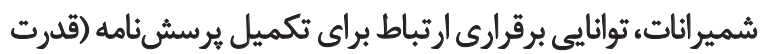

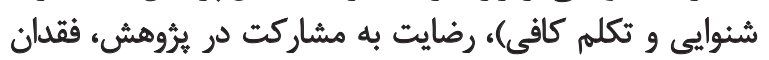

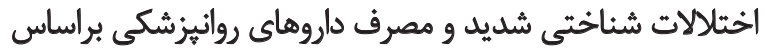
مندرجات برونده يزشكى بيمار. 
آسايشكاههاي شهرستان شميرانات رابطه معنادار وجود دارد

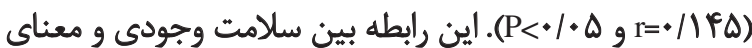

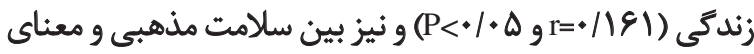

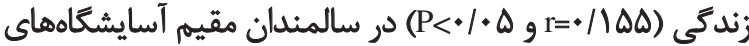
شهرستان شميرانات معنى دار است.

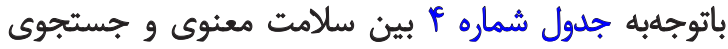

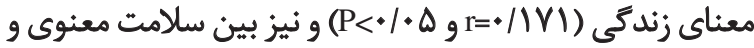

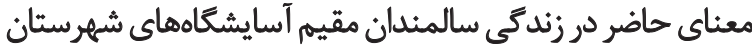

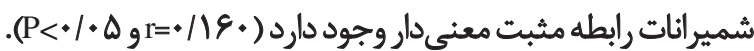

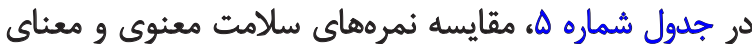
زندگى در دو جنس زن و مرد نشان داده شده است.

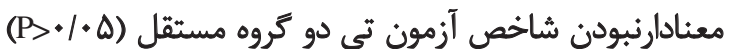

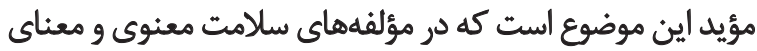

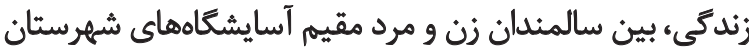

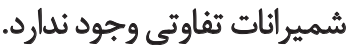

رابطه سلامت معنوى و معناى زندكى با تحصيلات در جدول

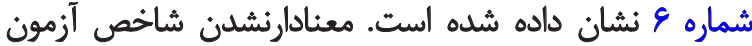

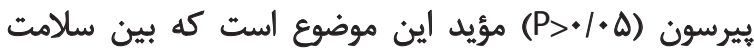

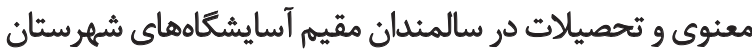

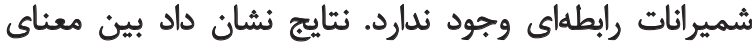

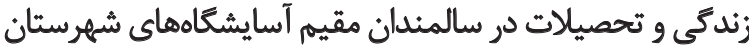

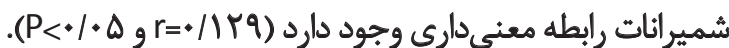

بحث

نتّايج اين مطالعه نشان داد نمره سلامت معنوى در بيشتر

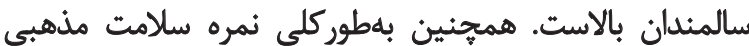

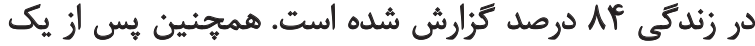

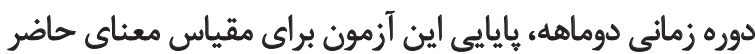

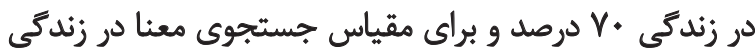

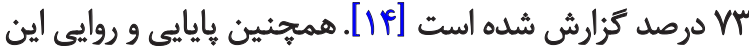

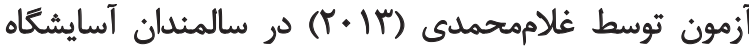

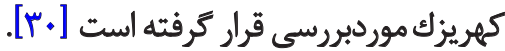

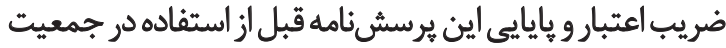

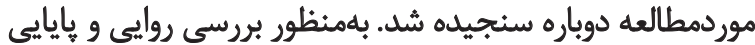

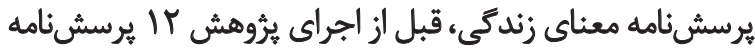

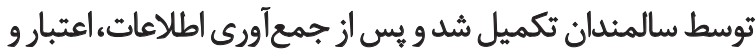

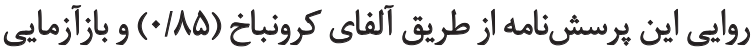

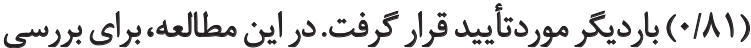

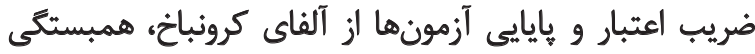

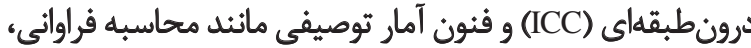

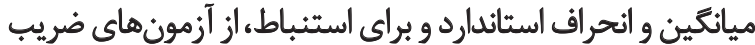
همبستكى بيرسون و تى مستقل استفاده شد.

ياثتهها

در اين تحقيق،

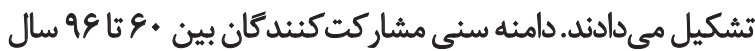

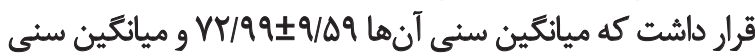

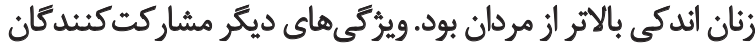

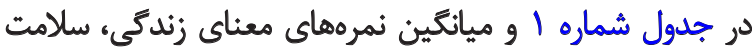

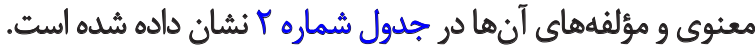

همانطوركه در جدول شماره ب ديده ميشود، بين ميانكين

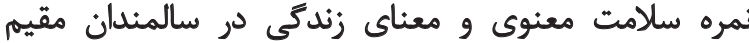

جدول ا. توزيع فراواني متغيرهاي جمعيتشناختي در سالمندان مقيم آسايشعامهاي شهرستان شميرانات.

\begin{tabular}{|c|c|c|c|}
\hline مرصد & تعداد & \multicolumn{2}{|c|}{ مثتغير } \\
\hline$m p / \Lambda$ & iv & مرد & \multirow{2}{*}{ جنسيث } \\
\hline so/r & irr & ن & \\
\hline 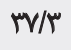 & ve & بيىسواد & \multirow{4}{*}{ تحصيلات } \\
\hline$m / q$ & ED & ابتدايي و راهنمايي & \\
\hline ro & Q) & دييلم & \\
\hline$\Delta / q$ & ir & ليسانس و بالاتر & \\
\hline rile & pr & بلون همسر & \multirow{2}{*}{ تأهل } \\
\hline VNF & 18. & مثتأهل & \\
\hline NA & M & خوب & \multirow{3}{*}{ وضعيت اقتصادى } \\
\hline $8 \cdot 11$ & infe & هتوسط & \\
\hline$r \cdot \mu$ & er & ضعيف & \\
\hline
\end{tabular}


جدول r. ميانكين ثمرههاى معناى زندگى و سلامت معنوى و مؤلفهاى آنها در سالمندان مقيم آسايشكاههاى شهرستان شميرانات.

\begin{tabular}{|c|c|c|}
\hline انحرافمعيار & مياتكين & مثغير \\
\hline$m / q \mu$ & $\Delta \& / . r$ & معناى زندكى \\
\hline $1 / 19$ & $r V / \cdot 1$ & جستجوى معناي زندكى \\
\hline T/AF & $r q /+r$ & معناى حاضر در زندكى \\
\hline IT/EA & $V / q \varepsilon$ & سلامت معنوى \\
\hline$V / \mu \Lambda$ & $r \mid / \wedge l$ & سلامت وجودى \\
\hline$V / T F$ & $r \Delta / r$ & سلامت مذهبى \\
\hline
\end{tabular}

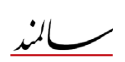

جدول با. ميزان همبستكى معناى زندكى با سلامت معنوى و مؤلفههاى آن در سالمئدان مقيهم آسايشكاههاى شهرستان شميرانات.

\begin{tabular}{|c|c|c|}
\hline \multicolumn{2}{|c|}{ معناى زندكى } & \multirow{2}{*}{ نام متغير } \\
\hline $\mathbf{p}$ & $\mathbf{r}$ & \\
\hline$+1 \cdot r$ &.$/ 1 F a$ & سلامت معنوى \\
\hline$+1+r$ &. $\mid 181^{\circ}$ & سلامت وجودى \\
\hline$+1 \cdot r$ &.$/ 1 \Delta \Delta^{\circ}$ & سلامت مذهبى \\
\hline
\end{tabular}

il

جدول F. همبستكى سلامت معنوى و مؤلفههاي معناى زندكى در سالمندان مقيم آسايشكادهاى شهرستان شميرانات.

\begin{tabular}{|c|c|c|}
\hline \multicolumn{2}{|c|}{ سلامت معنوى } & \multirow{2}{*}{ نام متغير } \\
\hline $\mathbf{p}$ & $\mathbf{r}$ & \\
\hline $.1 \cdot r$ &.$/ 1 f a$ & معناى زندكى \\
\hline.$/ 1$ & $. / 1 n)^{*}$ & جستجوى معناي زندكى \\
\hline $.1+r$ &.$/ 18$. & معناي حاضر در زندكى \\
\hline
\end{tabular}

닌

جدول ه. آزمون تى دو كروه مستقل براى بررسى تفاوت سلامت معنوى و معناى زندكى سالمثلدان زن و مرد در سالمثدان مقيم آسايشعاههاى شهرستان شميرائات.

\begin{tabular}{|c|c|c|c|c|}
\hline \multirow{2}{*}{ p } & \multirow{2}{*}{$\mathbf{T}$} & مرد & زن & \multirow{2}{*}{ متغير } \\
\hline & & هيانغين ثانحر افمعيار & ميانئين+|نحرافمعيار & \\
\hline . MAFA &. $\mid V \& 1$ & W/AVEVT/+F & $\| r / \cdot V \pm V T / F \Delta$ & سلاذهت معنوى \\
\hline $.18+1$ &.$(\Delta) F$ & $r / Q \curlyvee \pm \Delta \Delta / A \rho$ & r/q $\pm \Delta \& / / P$ & معناى زندكى \\
\hline
\end{tabular}

Uـ

به مسائل و تغييرات دوران سالمندى و معنابخشيدن به زندكى، ماهي،

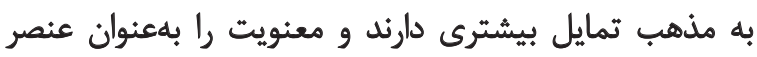

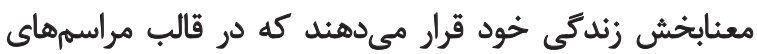

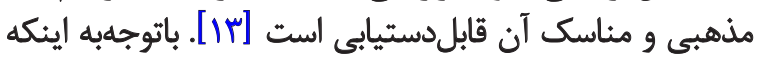

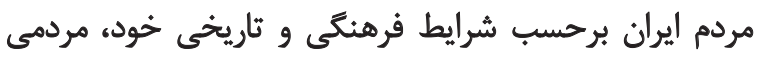

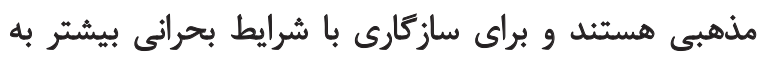

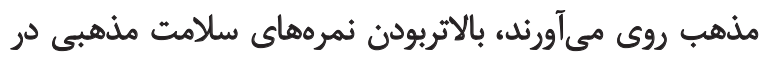

سالمندان نسبت به سلامت وجودى آنها بالاتر است. اين نتيجه

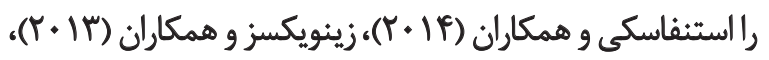

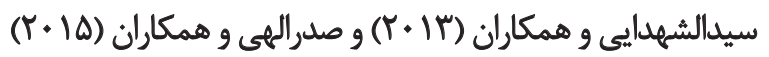

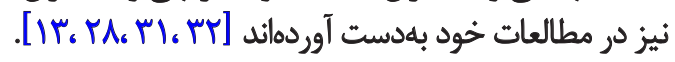
اين نتايج مى تواند ناشى از آن باشد كه با افزايش سن توجه به

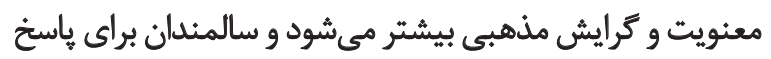


جدول و. ميزان همبستكى بين سلامت معنوى و معناي زندكى با تحصيلات در سالمندان مقيم آسايشكاههاي شهرستان شميرانات.

\begin{tabular}{|c|c|c|}
\hline \multicolumn{2}{|c|}{ تحصيلات } & \multirow{2}{*}{ متغير } \\
\hline $\mathbf{P}$ & $\mathbf{r}$ & \\
\hline .101 & $* n$ & سلامت معنوى \\
\hline H.r &.$/ 1 r q^{*}$ & معناى زندكى \\
\hline
\end{tabular}

L

قرار مى دهد. سالمندان سعى مي كنند عقايد و تجربيات معنوى

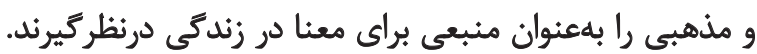

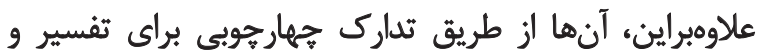

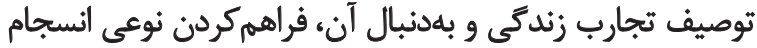

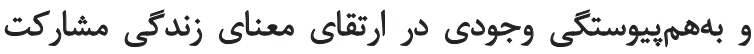

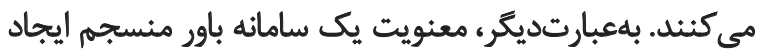

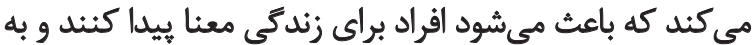

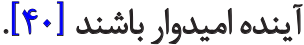

افراد سالمندى كه از سلامت معنوى برخوردارند، اين توانايى

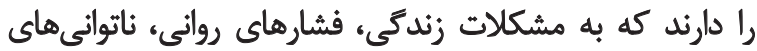

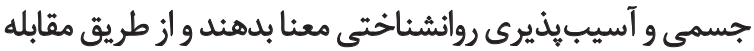

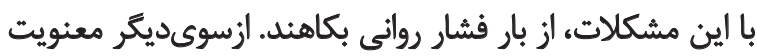

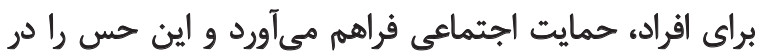

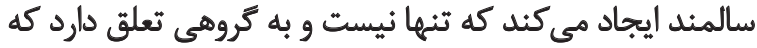

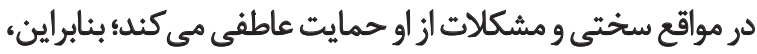

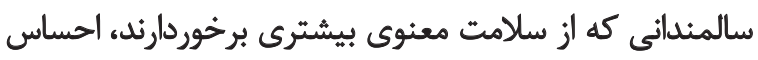
هدفمندى و معناى زندكى بيشترى دارند.

نتايج اين ثروهش نشان داد بين سلامت وجودى و معناى

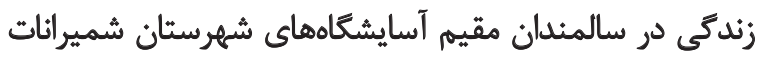

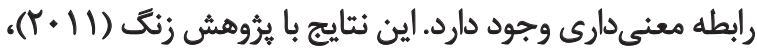

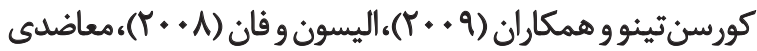

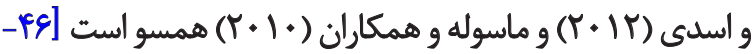

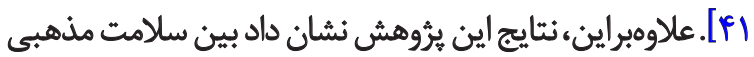

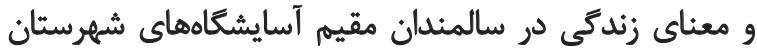

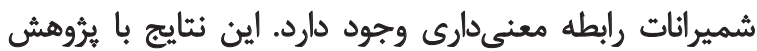

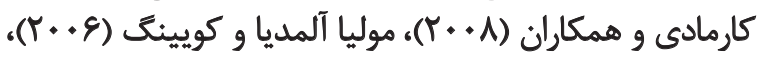

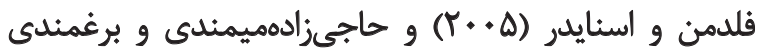

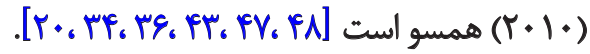

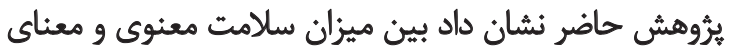

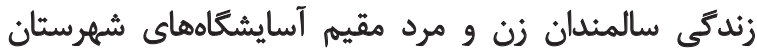

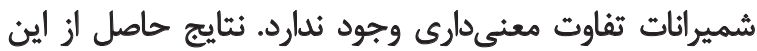

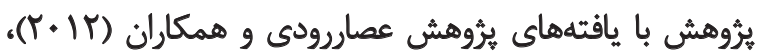

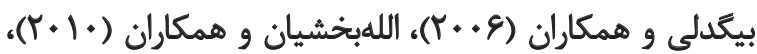

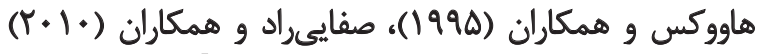

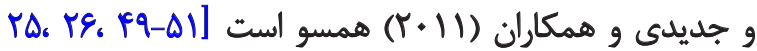

\section{آنان يافتهاي نسبتًا بيشبينى يذير و قابل توجيه است.}

نتايج اين يثروشش نشان داد بين سلامت معنوى و معناى

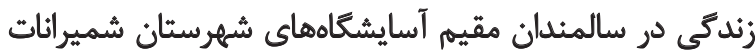

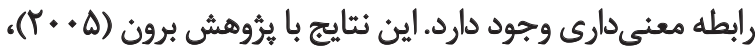

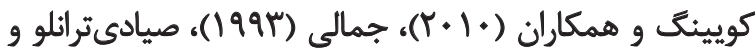

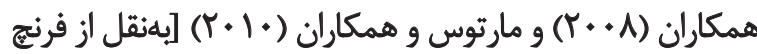

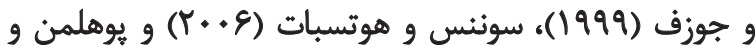

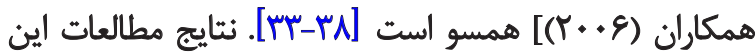

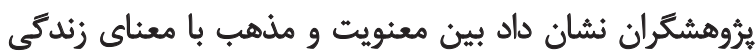

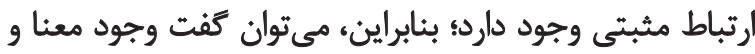

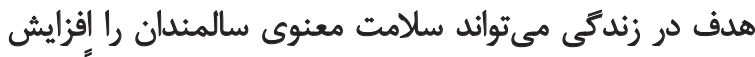

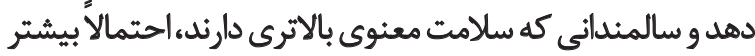
معناو هدف زندكى خود را يافته باشند.

در تبيين نثّايج حاصل از اين يثروهش مي توان كفت براساس نظريه

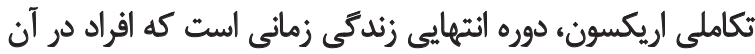

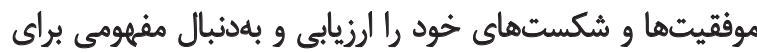

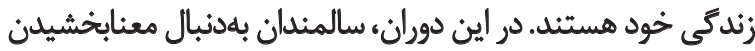

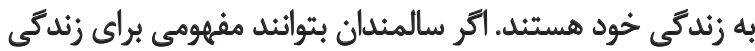

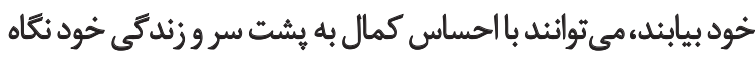

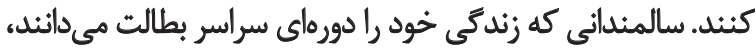

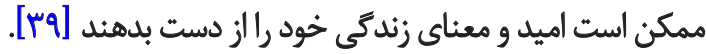

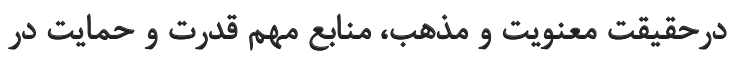

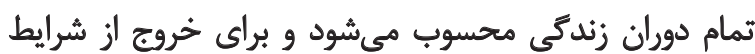

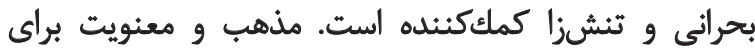

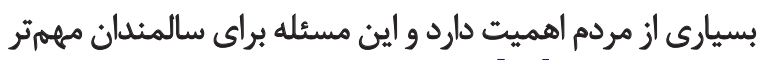

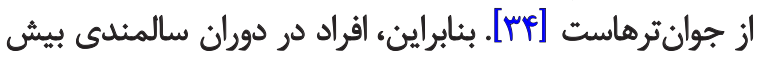

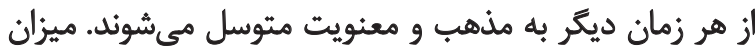

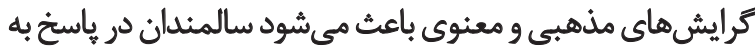

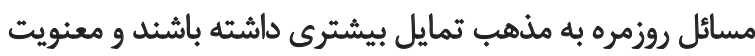

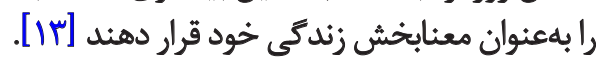

معنويت و سلامت معنوى از جمله عوامل يبيشبينى كنينده معنا

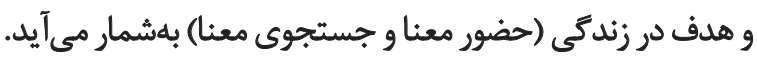

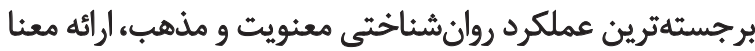

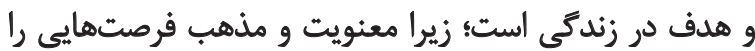

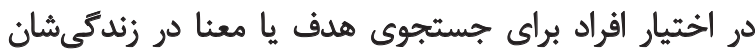


حاضر، ميتوان به متخصصان سلامت روان و افراد فعال در حوزه

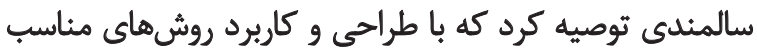

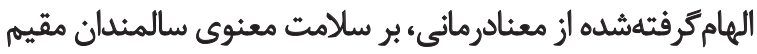

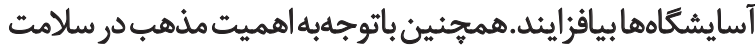

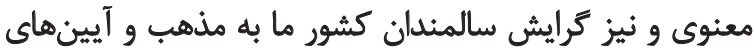

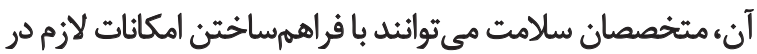

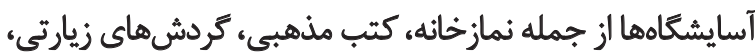

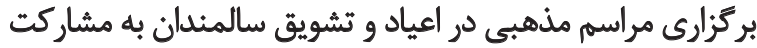
در امور خير سلامت معنوى سالمندان را تقويت كنئد.

انجام تحقيقات مشابه روى سالمندانى كه در جمع خانواده

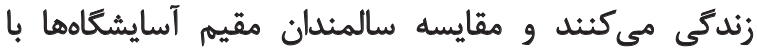

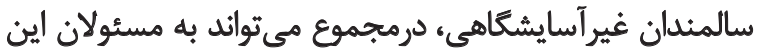

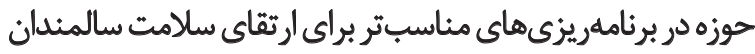

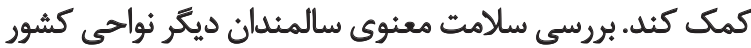

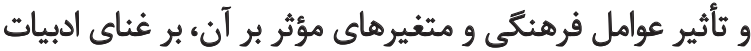
علمى در اين زمينه خواهد افزوني

محدوديتها

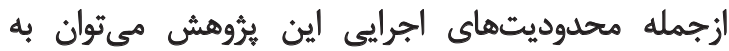

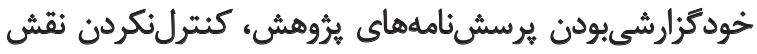

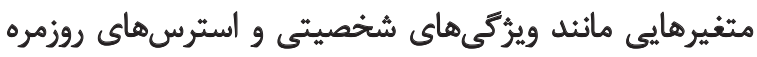

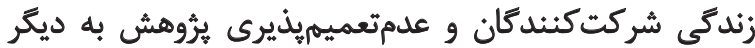
كروههاى سالمندان اشاره كرد.

$$
\text { تشكر و قبدردانى }
$$

دريايان، از حمايتهاي بى بدريغ مسئولان و كاركنان

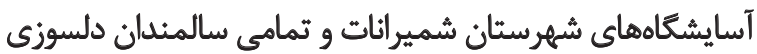

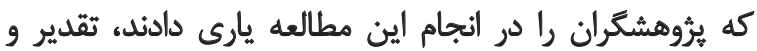

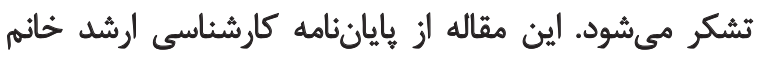

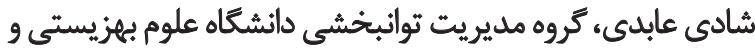
توانبخشى تهران كرفته شده است.

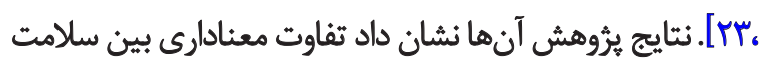

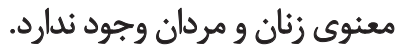

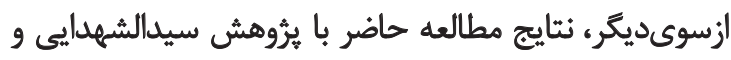

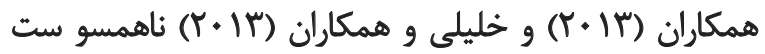

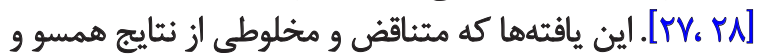

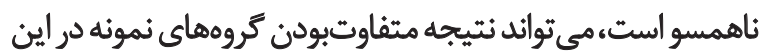

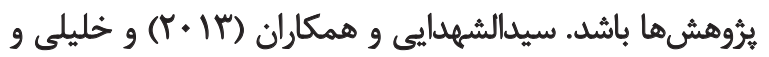

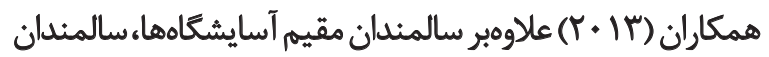

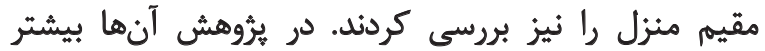

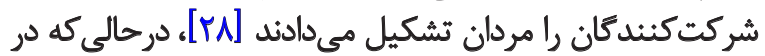

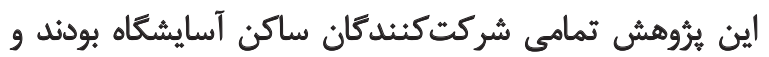

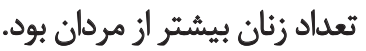

تتايج حاصل از اين يُروهش نشان داد بين سلامت معنوى و

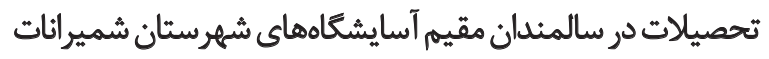

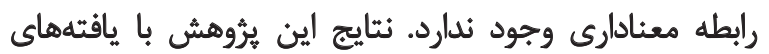

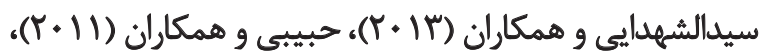

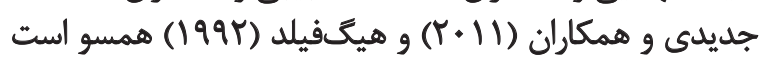

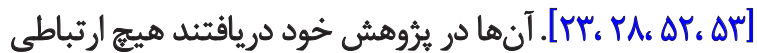

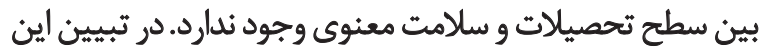

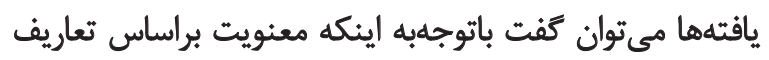

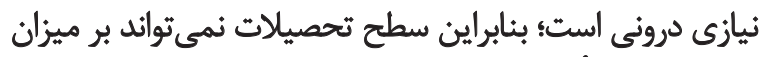
معنويت افراد تأثير تئار باشد.

علاوهبراين، نتايج اين يُؤوهش نشان داد بين معناي زندكى و و

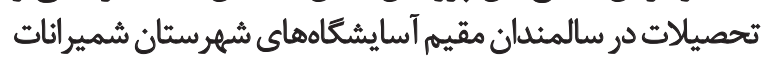

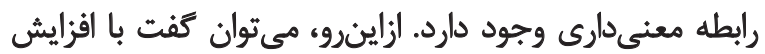

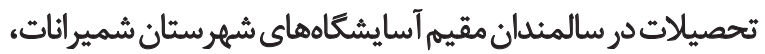

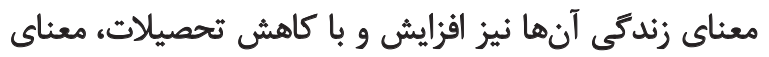

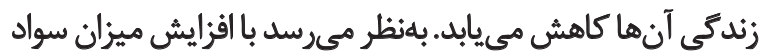

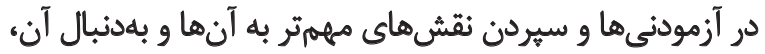

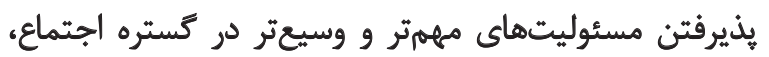

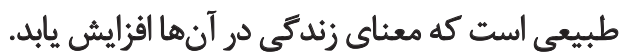

\section{نتيجه تيرى نهايى}

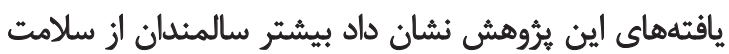

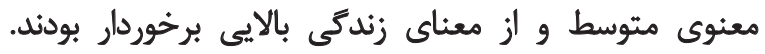

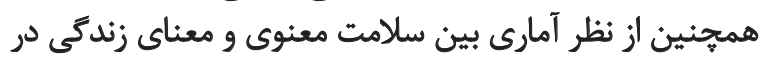

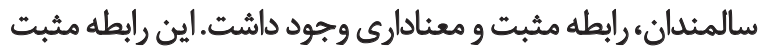

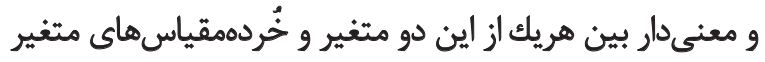

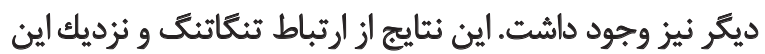
دو متغير و مؤلفههاى آن با يكديخر حكايت دارد.

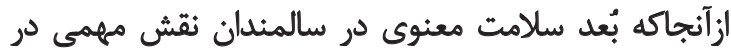

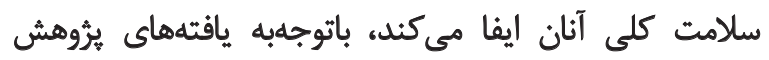




\section{References}

[1] Hofmanwold G. Nursing in elderly [HA. Abedi, M. Shahriari, N. Alimohammadi, A. Yazdannik, H. Yousefi, Persian trans]. Isfahan: Orroj Publication; 2003.

[2] World Health Organization. Partners in life skills education. Geneva: World Health Organization; 1999.

[3] Statistical Center of Iran. [Census 2006 (Persian)]. 2011. Tehran:Statistical Center of Iran; 2006.

[4] King LA, Hicks JA, Krull JL, Del Gaiso AK. Positive affect and the experience of meaning in life. Journal of Personality and Social Psychology. 2006; 90(1):179-96.

[5] Hedayati MM, Khazaei MM. An investigation of the relationship between depression, meaning in life and adult hope. ProcediaSocial and Behavioral Sciences. 2014; 114:598-601.

[6] Baumeister RF. Meanings of life. New York: Guilford Press; 1991.

[7] Lavasani M, Ejei J, Mohammadi F. The relationship between meaning of life and optimism with subjective well-being. Journal of Psychology. 2013; 17(1):3-17.

[8] Karimi-Ghoddusi Z. [God and the meaning of life in a perspective of Allame Tabataba'i and Ted Metz (Persian)]. Tehran :Tarbiyat Moallem Press; 2009.

[9] Martiny J. Suicide ideation in the elderly. Journal of Psychiatric Times [Internet]. 2004 [2004 March 1]. Available from: http:// www.psychiatrictimes.com/articles/suicide-ideation-elderly.

[10] Kretzer K, Evelo AJ, Durham RL. Lessons learned from a study of a complementary therapy for self-managing hypertension and stress in women. Holistic Nursing Practice. 2013; 27(6):336-43.

[11] Ghobari Bonab B, Raghebian R. [Children, teenager and spirituality (Persian)]. Tehran: Yastorun Publication; 1997.

[12] Boivin MJ, Kirby AL, Underwood LK, Silva H. Review of the spiritual well-being scale. In: Hill JPC, Hood RW, editors. Measures of Religiosity. Birmingham, A.L.: Religious Education Press; 1999, p. 382-85.

[13] Zenevicz L, Moriguchi Y, Madureira VS. A religiosidade no processo de viver envelhecendo. Revista da Escola de Enfermagem da USP. 2013; 47(2):433-39.

[14] Steger MF, Frazier P, Oishi S, Kaler M. The meaning in life questionnaire: assessing the presence of and search for meaning in life. Journal of Counseling Psychology. 2006; 53(1):80-93.

[15] Bishop AJ, Randall GK, Merten MJ. Consideration of forgiveness to enhance the health status of older male prisoners confronting spiritual, social, or emotional vulnerability. Journal of Applied Gerontology. 2014; 33(8):998-1017.

[16] Ghahramani L, Nazari M, Mousavi M. [Improvement of quality of life in elderly men in kahrizak nursing home based on educational intervention (Persian)]. Journal of Knowledge and Health. 2009; 4(2):18-23.

[17] Ebrahimi A. The relationship between depression and religious Attitude and Performances in Adults Isfahan 1998. Journal of Research in Medical Sciences. 2003; 8(1):94-95.

[18] Pourebrahim T, Rasouli R. [Effects of group logotherapy on decreasing depression and increasing meaning in life on older adults resident in sanatorium (Persian)]. Journal of Applied Psychology. 2008; 2(4):673-85.

[19] Ishaghi R, Mahmoudian SA, Asgarian R. [Effect of faith-based education on physical activity on the elderly (Persian)]. Iranian Journal of Medical Education. 2011; 10(5):1281-288.

[20] Hadjizadeh Meimandi MD, Barghamadi M. [The study of the relationship between religious beliefs performance and life satisfaction among the elderly (Persian)]. Iranian Journal of Ageing. 2010; 5(1):87-94

[21] Brown C. Professional social worker and anglican priest. London: Jessica Kingsley; 2005.

[22] Farhadi M, Ahmadi Tahoor Soltani M, Ramezani V, Gharehkhani A. [Elderly mental health: the role of spiritual well-being and hope (Persian)]. Journal of Research in Psychological Health. 2009; 3(2):43-50

[23] Jadidi A, Farahaninia M, Janmohammadi S, Haghani H. [The relationship between spiritual well-being and quality of life among elderly people residing in Kahrizak senior house (Persian)]. Iran Journal of Nursing, 2011; 24(72):48-56.

[24] Ju H, Shin JW, Kim CW, Hyun MH, Park JW. Mediational effect of meaning in life on the relationship between optimism and well-being in community elderly. Archives of Gerontology and Geriatrics. 2013; 56(2):309-13.

[25] Osarrodi A, Golafshani A, Akaberi S. [Relationship between spiritual well-being and quality of life in nurses (Persian)]. Journal of North Khorasan University of Medical Sciences. 2012; 3(4):7988.

[26] Bigdeli E, Karimzadeh S. [The effect of factors causing stress on mental health nurses in Semnan (Persian)]. Semnan University of Medical Sciences Journal (Koomesh). 2006; 8(2):21-26.

[27] Khalili F, Sum S, Asayesh H. [Spiritual health among Isfehanian elderly people (Persian)]. Iranian Journal of Ageing. 2013; 8(1):16-23.

[28] Saydshohadai M, Heshmat Sh, Seidfatemi N, Haghani $\mathrm{H}_{\text {, }}$ Mehrdad N. [The spiritual health of seniors living in sanitarium and home residents (Persian)]. Iran Journal of Nursing. 2013; 26(81):11-20.

[29] Seyyed-Fatemi N, Rezaei M, Givari A, Hosseini F. [Prayer and spiritual well-being in cancer patients (Persian)]. Payesh. 2006; 5(4):259-304.

[30] Gholam Mohammadi H. [Effectiveness of religious education based on the Quran, Hadith and Islamic narrations on the meaning of life and hope in the elderly in Kahrizak care center of the elderly (Persian)] [Msc. thesis]. Tehran: University of Social Welfare anf Rehabilitation Science; 2013.

[31] Sadrollahi A, Khalili Z. [Spiritual Well-being and associated factors among the elderly population in Kashan (Persian)]. Journal of Geriatric Nursing. 2015; 1(2):94-104

[32] Stefanaki IN, Shea S, Linardakis M, Symvoulakis EK, Wynyard $\mathrm{R}$, Lionis C. Exploring the association of sense of coherence, and spiritual and religious beliefs in a rural population group on the island of Crete, Greece. The International Journal of Psychiatry in Medicine. 2014; 47(3):207-30.

[33] Khakshoor F, Qobari Bonab B, Shahabizadeh F. [The role of conception of God and religious identity in the meaning of life (Persian)]. Psychology and Religion, 2013; 2(6):44-58. 
[34] Koenig HG. Religion, spirituality, and health: the research and clinical implications. ISRN Psychiatry. 2012. doi: $10.5402 / 2012 / 278730$

[35] Martos T, Thege BK, Steger MF. It's not only what you hold, it's how you hold it: dimensions of religiosity and meaning in life. Personality and Individual Differences. 2010; 49(8):863-68.

[36] Koenig HG. Spirituality, wellness, and quality of life. Sexuality, Reproduction and Menopause. 2004; 2(2):76-82.

[37] Jamali F. [The relationship between religious attitudes, Sense of meaning fulness of life and mental health among University students in Tehran (Persian)] [MA thesis]. Tehran: Alzahra University; 1993.

[38] Sayyadi Turanlu H, Jamali R, Mirghafuri SH. [The Relationship between belief to teachings of islam religion and emotional intelligence of students (Persian)]. A Research Quarterly in Islamic Theology (Kalam) and Religious Studies. 2008; 3(11):45-51.

[39] Capps D. The verbal portrait: Erik H. Erikson's contribution to psychoanalytic discourse. Journal of Religion and Health. 2011; 50(4):880-98.

[40] Seligman ME. Authentic happiness: using the new positive psychology to realize your potential for lasting fulfillment. New York: Simon and Schuster; 2004.

[41] Moazedi K, Asadi A. [Mental health status in the Quran (Persian)]. Journal of Ardabil University of Medical Sciences. 2012; 12(1):85-96.

[42] Reza Masoule Sh, Sheikhol Eslami F, Khodadadi N, Yazdani MA. [Study the role of the religious beliefs on general health status of the members of pensioners association of Guilan University of Medical Sciences-Rasht (Persian)]. Holistic Nursing and Midwifery Journal. 2010; 20(1):22-26.

[43] Carmody J, Reed G, Kristeller J, Merriam P. Mindfulness, spirituality, and health-related symptoms. Journal of Psychosomatic Research. 2008; 64(4):393-403.

[44] Corsentino EA, Collins N, Sachs-Ericsson N, Blazer DG. Religious attendance reduces cognitive decline among older women with high levels of depressive symptoms. Journals of Gerontology Series A: Biological Sciences and Medical Sciences. 2009; 64(12):1283-289.

[45] Ellison CG, Fan D. Daily spiritual experiences and psychological well-being among US adults. Social Indicators Research. 2008; 88(2):247-71.

[46] Zeng Y, Gu D, George LK. Association of religious participation with mortality among Chinese old adults. Research on Aging. 2011; 33(1):51-83.

[47] Feldman DB, Snyder CR, Hope and the meaningful life: theoretical and empirical associations between goal-directed thinking and life meaning. Journal of Social and Clinical Psychology. 2005; 24(3):401-21.

[48] Moreira-Almeida A, Koenig HG. Retaining the meaning of the words religiousness and spirituality: a commentary on the WHOQOL SRPB groups "A cross-cultural study of spirituality, religion, and personal beliefs as components of quality of life" (62: 6, 2005, 1486-1497). Social Science \& Medicine. 2006; 63(4):843-45.

[49] Allahbakhshian M, Jaffarpour M, Parvizy S, Haghani H. [A survey on relationship between spiritual wellbeing and quality of life in multiple sclerosis patients (Persian)]. Zahedan Journal of Research in Medical Sciences, 2010; 12(3):29-33.

[50] Hawks SR, Hull ML, Thalman RL, Richins PM. Review of spiritual health: definition, role, and intervention strategies in health promotion. American Journal of Health Promotion. 1995; 9(5):371-78.

[51] Safayi Rad I, Karimi L, Shomoossi N, Ahmadi Tahour M. [The relationship between spiritual well-being and mental health of university students (Persian)]. Quarterly Journal of Sabzevar University of Medical Sciences. 2010; 17(4):274-80.

[52] Habibi A, Savadpour MT. [Spiritual well-being in cancer patients under chemotherapy (Persian)]. Journal of Health and Care. 2011; 13(3):16-21.

[53] Highfield MF. Spiritual health of oncology patients: nurse and patient perspectives. Cancer Nursing. 1992; 15(1):1-8. 